\title{
Temperature Prediction of Power Cable Joint Based on LS-SVM Optimized by PSO
}

\author{
Bang-Le HE \\ State Grid Shanghai Cable Company, 200072 Shanghai, China
}

\begin{abstract}
The temperature of high-voltage cable has a great significance to reflect the operation status, and the accurate prediction of the joint temperature can improve the safe operating level of the wire. This paper points out a temperature prediction model based on least squares support vector machine (LS-SVM) to forecast short-term cable joint temperature. This paper also conducts a test on a Shanghai $110 \mathrm{kV}$ cable line with its joint's history temperature, environmental temperature and humidity, the wire core/sheath current ratio data, and the particle swarm optimization algorithm (PSO) can be adapted to optimize model parameter standardization and regularization parameter dynamically. The results prove that method can predict the temperature of cable joint with high prediction accuracy and also provide a reliable basis for cable temperature detection and early warning system.
\end{abstract}

\section{Introduction}

The power cable transmission system can beautify city's appearance and largely save urban land resources, completely meet the needs of resources saving and environment-friendly ${ }^{[1]}$. Power cables are widely used in the urban grid and its demand is growing fast. According to the field operation experience, the weakness of the cable system is the cable joint and there are over $90 \%$ of the cable failure occurs in the position of the cable joints ${ }^{[2]}$. The internal defects of cable joint will cause electric field concentration, local temperature increase $\mathrm{e}^{[3]}$ and electric-thermal breakdown on power cable insulation ${ }^{[4]}$, if its temperature is more than $137^{\circ} \mathrm{C}$, and finally hardly damage the safe operation of power grid. Although joint temperature can properly reflect the operation situation of cable joint, existing cable monitoring system for cable joint temperature can only achieve real-time data acquisition without forecast. Therefore, it's necessary to forecast the temperature of the joint, prognosis the insulation level of the cable joint and detect fault in time for the temperature monitoring system's warning foundation.

There is nonlinear and random relationship between cable joint temperature and environmental temperature, humidity and wire core current. Literature [5] use the coefficient of the first-order and second-order adaptive optimization predict group legal and literature [6] with the method of generalized regression neural network to forecast, but there is weakness on high requirement to the sample data and convergence insufficiency. SVM (Support Vector Machines) regression model which follows the principle of risk minimization can effectively solve the practical problems such as small sample, nonlinearity and high latitudes ${ }^{[7]}$. SVM has been successfully applied to short-term load forecasting, wind power prediction and wind velocity prediction ${ }^{[8]}$. But SVM is long and computationally intensive ${ }^{[9]}$. LS-SVM (Least Squares Support Vector machines) is the improved algorithm of SVM ${ }^{[10]}$, it can convert quadratic programming problem into solving linear equations, reduce the computational complexity, and reduce convergence time when forecasting. Like SVM, LSSVM takes the kernel parameters and penalty parameters by experience, and there are too many human factors ${ }^{[1]}$. So, this approach requires intelligent optimization of parameters.

In this article, LS-SVM is firstly adapted to establish joint temperature prediction model with environment temperature, environment humidity, sheath/wire core current ratio and the duration cable joint temperature as the training sample. In order to improve the prediction accuracy, PSO (Particle Swarm Optimization) is used to dynamically optimize the regularization parameters $C$ and standardized parameters $\sigma$ of LS-SVM to construct a PSO-LSSVM prediction method. A 110kv cable line's termination joints in Shanghai have been tested and the prediction results show that this method can properly forecast the cable joint temperature with high accuracy and even provide reliable judgment for cable temperature detection and pre-warning system.

\section{A prediction method of LS-SVM}

The basic idea of SVM's data regression is that influencing factor $x_{i}$, which is closely related to the number of influencing factors is defined as input, forecast expectations $y_{i}$ is defined as output and the

\footnotetext{
* Corresponding author: hebangle@sina.com
} 
nonlinear mapping $\phi(x)$ from the input space is mapped to high-dimensional feature space $^{[7]}$, its nonlinear relationship defined as:

$$
y(x)=(\omega, \phi(x))+b
$$

where $\phi(x)$ is defined as the nonlinear mapping, $\omega \in R^{d}$ is defined as the weight vector and $b \in R$ is defined as the deviation value. There are $l d$ dimensional data point $\left(x_{i}, y_{i}\right), \quad i=1, \ldots, l$ and $x_{i} \in R^{d}, y_{i} \in R$ and quadratic norm of error is chosen as the loss function, according to the risk minimization principle, LS-SVM optimization model can be expressed as

$$
\left\{\begin{aligned}
\min & \frac{1}{2}\|\omega\|^{2}+\frac{1}{2} C \sum_{i=1}^{l} e_{i}^{2} \\
\text { s.t. } & \omega^{T} \phi\left(x_{i}\right)+b+e_{i}=y_{i}, i=1,2, \ldots, l
\end{aligned}\right.
$$

In equation (2), $e_{i}$ is defined as error, and $C$ is regularization parameter, which controlling the degree of punishment of error.

$$
\min L=\frac{1}{2}\|\omega\|^{2}+\frac{1}{2} C \sum_{i=1}^{l} e_{i}^{2}-\sum_{i=1}^{l} \lambda_{i}\left(\omega^{T} \phi\left(x_{i}\right)+b+e_{i}-y_{i}\right)
$$

By the KKT conditions, $\frac{\partial L}{\partial \omega}=0, \frac{\partial L}{\partial b}=0, \frac{\partial L}{\partial e_{i}}=0$, $\frac{\partial L}{\partial \lambda_{i}}=0$, and

$$
\left\{\begin{array}{l}
\frac{\partial L}{\partial \omega}=0 \rightarrow \omega=\sum_{i=1}^{l} \lambda_{i} \phi\left(x_{i}\right) \\
\frac{\partial L}{\partial b}=0 \rightarrow \sum_{i=1}^{l} \lambda_{i}=0 \\
\frac{\partial L}{\partial e_{i}}=0 \rightarrow \lambda_{i}=C e_{i}, i=1,2, \ldots, l \\
\frac{\partial L}{\partial \lambda_{i}}=0 \rightarrow \omega^{T} \phi\left(x_{i}\right)+b+e_{i}-y_{i}=0
\end{array}\right.
$$

delete $\omega$ and $e$, there is system of linear equation:

$$
\left[\begin{array}{cc}
0 & U^{T} \\
U & \Omega+C^{-1}
\end{array}\right]\left[\begin{array}{l}
b \\
\lambda
\end{array}\right]=\left[\begin{array}{l}
0 \\
Y
\end{array}\right]
$$

In equation ( 5$), \lambda=\left[\lambda_{1}, \lambda_{2}, \ldots \lambda_{l}\right]^{T}, U=[1,1, \ldots, 1]^{T}$, $Y=\left[y_{1}, y_{2}, \ldots, y_{l}\right]^{T}$ is $l \times 1$ dimensional column vector, $\Omega \in R^{l \times l}$ is kernel function and $\Omega_{i j}=\phi\left(x_{i}\right)^{T} \phi\left(x_{j}\right)$.

A radial basis kernel function ${ }^{[12]}$ with good performance is chosen as LS-SVM kernel function, the form is

$$
K\left(x, x_{i}\right)=\exp \left(-\left\|x-x_{i}\right\|^{2} / \sigma^{2}\right)
$$

In equation (6), $x_{i}$ is the $i$ th center of radial basis function, and $\sigma$ is standard function, which determines the width of the center of the function.

$$
y=\sum_{i=1}^{l} \lambda_{i} K\left(x_{i}, x\right)+b
$$

\section{Using PSO optimize the parameters of LS-SVM}

During the prediction by LS-SVM, regularization parameter $C$, which is to control the degree of punishment and kernel function's normalizing parameter $\sigma$ should be given. Low ${ }_{C}$ will cause the big error and too much will cause poor promotion ability.

Too little $\sigma$ will cause excessive localize training and too much $\sigma$ will cause less training. Artificial selected parameters cannot guarantee the accuracy of forecasting model. To raise the forecasting precision of LS-SVM, this paper uses particle swarm optimizing algorithm for dynamic optimization.

Particle swarm algorithm has been widely applied in the field of optimization ${ }^{[13]}$. Parameter selection problem of support vector machines (SVM) can be regarded as global search problem of a given space by particle swarm optimization algorithm, specific steps are as follows:

STEP 1: The sample data are processed by normalization, initialization parameter $c_{1}, c_{2}, w, m$ and $N_{\max }$ of PSO, $C$ and $\sigma$ as a group of initialized particles. Among them, $c_{1}$ and $c_{2}$ are defined as acceleration constant, $w$ is defined as the inertia weight, $m$ is defined as the population size, $N_{\max }$ is defined as the biggest evolution algebra, the $C$ (regularization parameter) and $\sigma$ (kernel parameter) are mapped to a group of particles.

STEP 2: $f\left(x_{i}\right)$ (the adaptive value of each particle) is calculated to compare the value of particle position, will be the $i$ th particle's current point is set to $p_{\text {ibest }}$ (optimal location) and the optimal particle is defined as $g_{\text {best }}$ (population optimal position).

STEP 3: Updating each particle's speed and position by eq. 8 and eq. 9 and generate $X(n)$ (new population).

$$
\begin{gathered}
v_{t \delta}=\omega v_{t \delta}+\chi_{1} \rho_{1}\left(\pi_{t \delta}-\xi_{t \delta}\right)+\chi_{2} \rho_{2}\left(\pi_{\gamma \delta}-\xi_{t \delta}\right) \\
x_{i d}=x_{i d}+v_{i d}
\end{gathered}
$$

STEP 4: Calculating the adaptive value of $X(n)$, new $p_{\text {ibest }}$ and $g_{\text {best }}$, making a comparison with historical $p_{\text {ibest }}$ and $g_{\text {best }}$, making a replacement if new parameters are better, otherwise making no change.

STEP 5: Determining whether the evolution algebra has reached $N_{\max }$ or precision is less than $\mathcal{E}$, if it has reached, then output optimal $C$ and $\sigma$, otherwise, let $n=n+1$ and turn to step 2 .

\section{Cable joint temperature prediction}

\subsection{Data vector}

To predict the temperature of the cable joint using the PSO-LSSVM algorithm, it is necessary to determine sample data such as temperature-dependent variables and historical temperature as input quantities to form training samples and test samples. The high-voltage power cable joints of $110 \mathrm{kV}$ and above are restored according to the cable structure and materials when the insulation is restored. The equivalent heat map of the joint can be 
equivalently represented by the equivalent heat path diagram of the cable body, as shown in Figure 1.

The thermal resistance of the insulation layer is related to the core current. The thermal resistance of the inner liner and the thermal resistance of the outer layer are equivalent to the thermal resistance of the metal sheath and the circulation of the sheath. The thermal resistance of the surrounding medium is related to the ambient temperature and the ambient humidity. Results Operating experience, cable joint temperature is related to ambient temperature, humidity, core current and sheath current. The sheath current is generated by the sheath induced voltage in the sheath loop, and there is a certain proportional relationship with the core current. Therefore, the ambient temperature, ambient humidity, sheath/core current ratio, and historical temperature of the cable joint are used as input samples.

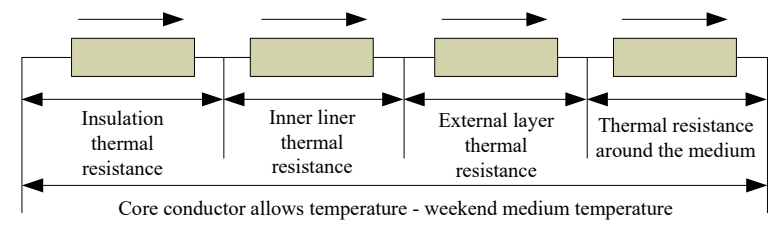

Fig. 1. Single core cable heat road diagram

All data can be obtained from the high-voltage cable operating status online monitoring system. The data is collated to form a data vector. Each data vector consists of 29 data of the average ambient temperature, the highest ambient temperature, the lowest ambient temperature, the ambient humidity, the sheath/core current ratio, and the measured temperature of the joint at the hour of the day, as shown in Figure 2. T1, .., T2 are the measured temperatures of the power connectors at the hour.

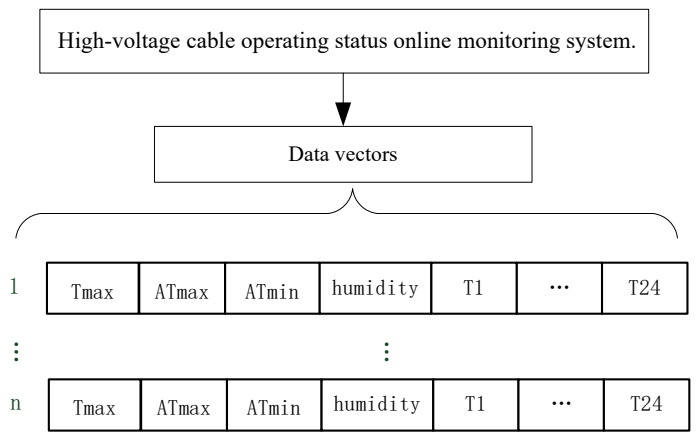

Fig. 2. On line monitoring system interface of high voltage cable

\subsection{Prediction steps and parameters selection}

This paper chooses MAPE (Mean Absolute Percentage Error) and MSE (Mean Square Error) which is commonly used in temperature prediction as evaluation standard.

RE-Relative Error:

$$
E_{\mathrm{RE}}=\frac{|L-\hat{L}|}{L} \times 100 \%
$$

MAPE-Mean Absolute Percentage Error:

$$
E_{\mathrm{MAPE}}=\frac{1}{n} \sum_{i=1}^{n}\left|\frac{L_{i}-\hat{L}_{i}}{L_{i}}\right| \times 100 \%
$$

In equation, $L$ and $\hat{L}$ is respectively actual and predicted temperature, $n$ is the number of temperature data.

At this point, the steps to forecast cable joint temperature by PSO-LSSVM have been set up, procedure is shown in figure 3.

Writing programs by Matlab tool, $m$ (the parameter of PSO algorithm) is set as $20, N_{\max }$ is set as $10, w$ is chosen in $[0.4,0.9]$. To balance the effect of random factors, $c_{1}$ and $c_{2}$ are both 2 in this paper.

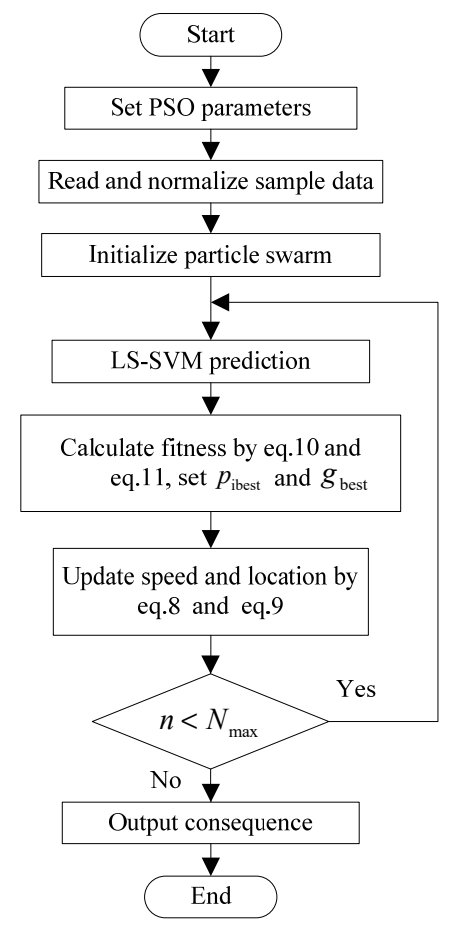

Fig. 3. Flow chart of PSO-LSSVM forecast

\section{Results}

In order to verify the prediction accuracy of the PSOLSSVM method, Experiment 1 (choosing the data from 2016.10.3 to 10.9 to predict the temperature of 10th day) and Experiment 2 (choosing the data from 10-3-016 to 10-17-2016 to predict the temperature of 16th day) simultaneously compared the prediction data of the PSOLSSVM and LSSVM methods.

Table 1. Comparison of model parameter optimization and average relative error

\begin{tabular}{|c|c|c|c|c|}
\hline Experiment & Method & $C$ & $\sigma$ & MAPE\% \\
\hline \multirow{2}{*}{1} & LS-SVM & 30 & 2 & 0.0572 \\
\cline { 2 - 5 } & PSO-LSSVM & 143.52 & 7.18 & 0.0553 \\
\hline \multirow{2}{*}{2} & LS-SVM & 30 & 2 & 0.0271 \\
\cline { 2 - 5 } & PSO-LSSVM & 7.0857 & 10 & 0.0210 \\
\hline
\end{tabular}

Table 1 shows both tests' optimization results and comparison value of average relative error, the LS-SVM parameters $C$ and $\sigma$ in both tests are respectively 30,2, 
the PSO-LSSVM parameters $C$ and $\sigma$ in first experiment is respectively the optimization of 143.52 and 7.18 , the PSO-LSSVM parameters $C$ and $\sigma$ in second experiment is respectively the optimization of 7.0857 and 10 .

Figure 4 shows the temperature curve of test 1 , the relative error as shown in figure 5 .

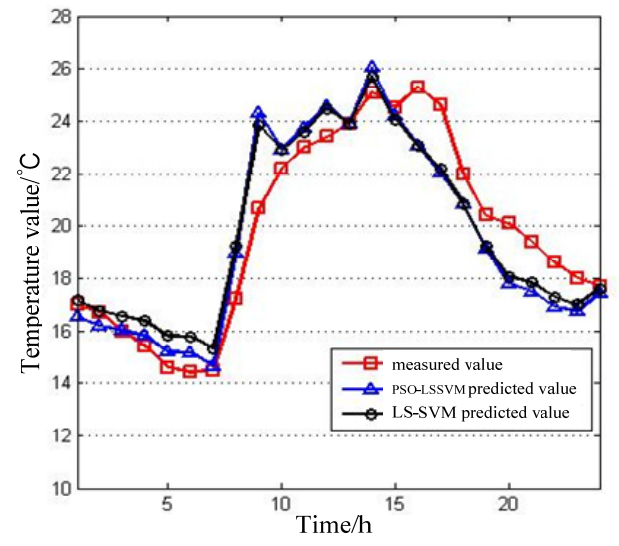

Fig. 4. Temperature curve of Experiment 1

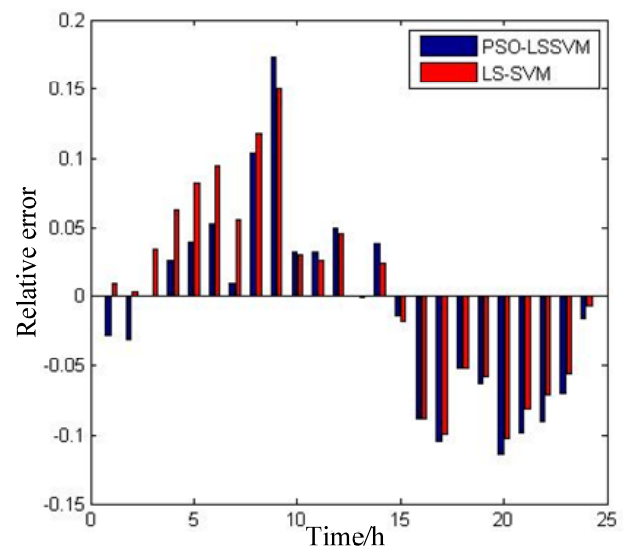

Fig. 5. Relative error of Experiment 1

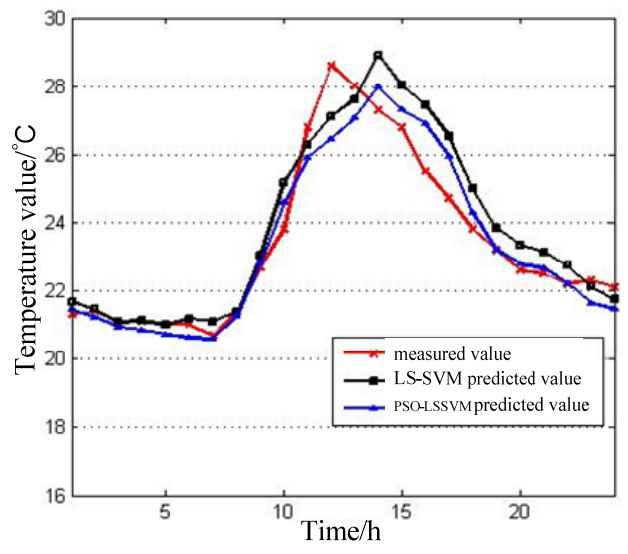

Fig. 6. Temperature curve of Experiment 2

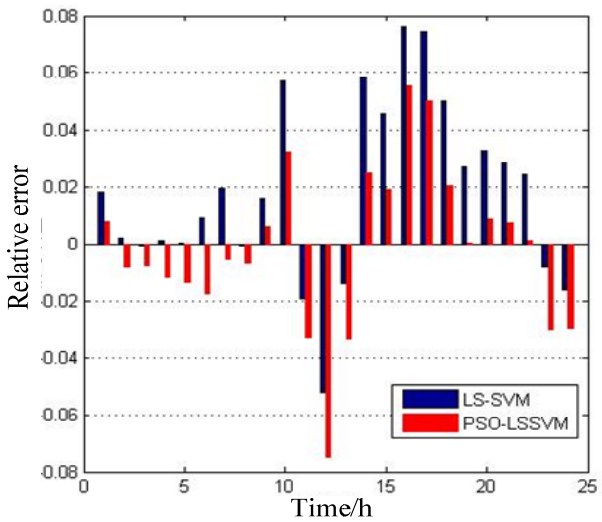

Fig. 7. Relative error of Experiment 2

Figure 6 is the predictive results of the experiment 2 , figure 7 for its relative error.

According to above mentioned data, the prediction of PSO-LSSVM is closer to the measured data and its relative error and average relative error is better than the prediction of LS-SVM. Thus, parameter optimization is effective.

The average relative error of experiment 1 is $0.0553 \%$, more than $0.021 \%$ of experiment 2 . This indicates that the size of the data samples will affect the forecasting result and reasonable selection of data sample is helpful to improve the prediction accuracy. The maximum temperature error of the experiment is $2.143{ }^{\circ} \mathrm{C}$, which occurred at 12 , at the same time, its absolute value of the relative error is $0.0748 \%$, which satisfies the requirement of forecast.

\section{Conclusion}

In this paper, PSO is adapted to dynamically optimize LS-SVM parameters. Joint history temperature, environment temperature, humidity, wire core/sheath current ratio is selected as the training sample to establish the cable joint temperature prediction model of PSO-LSSVM, which can improve the prediction performance and effectively predict the cable joint temperature. The size of data samples will affect the forecasting result and reasonable selection of data sample is helpful to improve the prediction accuracy. Experimental results show that this method has good convergence, higher precision and faster training speed, it can also provide reliable judgment to cable temperature detection and warning system. Thus, this method is of great value in engineering application.

\section{References}

1. Y.X. ZHOU, J.K. ZHAO, R. LIU, HVE, 40, 10 (2014)

2. Y.P. GAO, T.Y. TAN, K.P. LIU, HVE, 42, 8 (2016)

3. C. PENG, Y.J. ZHANG, W.N. QIN, HVE, 40, 7 (2014)

4. X.Q. GAO, Y. JIANG, J.H. LU, HVE, 2 , 3 (1997)

5. C.F. TIAN, Electric Wire \& Cable, 3, 4 (2015) 
6. W. XIAO, Q. HAN, W.T. ZHU, Electrotechnical Application, 13, 4 (2013)

7. V.N. VAPNIK, The nature of statistical learning theory(Tsinghua university press, Beijing, 2000)

8. B. XIAO, P. NIE, G. MU, AEPS, 12, 6 (2015)

9. X.X. ZHU, Z.H. HAN, Proceeding of the CSEE, 23, $6(2016)$

10. Y.P. GU, W.J. ZHAO, Z.S.WU, Proceeding of the CSEE, 30, 7 (2010)

11. X.Y. WU, J.H. HE, P. ZHANG, AEPS, 39, 6 (2015)

12. S S KEERHI, C J LIN, Neural Computation, 15, 23 (2003)

13. K.J. LI, Y.S. XU, B.G. WEI, HVE, 44, 8 (2018) 